\title{
Testing socioeconomic status and family socialization hypotheses of alcohol use in young people: A causal mediation analysis
}

\author{
David R. Foxcroft \\ Sarah J. Howcutt \\ Fiona Matley \\ Louise Taylor Bunce \\ Emma L. Davies (ㅁ)
}

Prevention Science Group, Oxford Brookes University, Oxford, UK

\section{Correspondence}

David R. Foxcroft, Prevention Science Group, Faculty of Health and Life Sciences, Oxford Brookes University, Headington Campus, Gipsy Lane, Oxford OX3 OLF, UK.

Email: david.foxcroft@brookes.ac.uk

\begin{abstract}
Introduction: The effect of socioeconomic status on adolescent substance abuse may be mediated by family socialization practices. However, traditional mediation analysis using a product or difference method is susceptible to bias when assumptions are not addressed. We aimed to use a potential outcomes framework to assess assumptions of exposure-mediator interaction and of no confounding of the results.

Method: We revisited a traditional mediation analysis with a multiple mediator causal mediation approach using data from 17,761 Norwegian young people (13-18 years), $51 \%$ female. Data were collected through a print questionnaire. Socioeconomic status was operationalized as parental education and employment status (employed or receiving welfare); drinking behavior as the frequency of alcohol consumption and frequency of intoxication in the past year; and socialization practices as general parenting measures, alcohol-related parental permissiveness, and parent drinking behavior.

Results: There was no consistent evidence of exposure-mediator interaction. Formal sensitivity analysis of mediator-outcome confounding was not possible in the multiple mediator model, and this analysis supported the hypothesis that socioeconomic status effects on adolescent substance abuse are fully mediated by family socialization practices, with apparently stronger effects in younger age groups observed in plots. Conclusion: We found that the effect of socioeconomic status on adolescent substance abuse was fully mediated by family socialization practices. While our analysis provides more rigorous support for causal inferences than past work, we could not completely rule out the possibility of unmeasured confounding.
\end{abstract}

\section{K E Y W O R D S}

alcohol, causal mediation, family socialization

\section{INTRODUCTION}

Socioeconomic status (SES) is associated with a range of health behaviors. In general, lower SES is associated with more risky behaviors and worse health outcomes. For example, the prevalence of the four main risk behaviors for noncommunicable diseases, unhealthy diet, smoking, sedentary activity, and excessive drinking, is typically higher in more deprived or lower status groups and communities, contributing to the social gradient in health (M. Marmot, 2005). To understand and intervene in these outcomes, it is important to understand what are the mechanisms through which SES influences health (M. G. Marmot, 2004). In young people, evidence suggests that SES is associated both with family socialization practices and with health risk behaviors, so understanding the causal relations between these three factors could contribute to understanding how SES influences health. A previous study (Pape et al., 2017) has used traditional mediation analysis to examine the

This is an open access article under the terms of the Creative Commons Attribution-NonCommercial License, which permits use, distribution and reproduction in any medium, provided the original work is properly cited and is not used for commercial purposes.

(C) 2022 The Authors. Journal of Adolescence published by Wiley Periodicals LLC on behalf of Foundation for Professionals in Services to Adolescents. 
relationship between these three factors. In this paper, we revisit this traditional mediation analysis using a more recent and more robust causal mediation approach.

If all around you is going to hell, the family really matters

(Michael Marmot, 2004)

Socialization can be regarded as multiple processes through which individuals develop characteristics (e.g., dispositions and behaviors) that are more or less healthy (Singh-Manoux \& Marmot, 2005). Intergenerational socialization is one process through which behaviors are transmitted across generations. This transmission occurs most prominently from parents to offspring, for example through bonding and social learning, which results in generational similarities in dispositions and behaviors. Studies show that particular parenting and family practices are associated with child risky behaviors, including smoking, drinking, and drug use (Cablova et al., 2014; Foxcroft \& Lowe, 1991, 1995, 1997). A theoretically informed perspective on family socialization practices in the context of adolescent substance use suggests four main domains: family love and support, parental control and monitoring, parental attitudes to adolescent substance use, and parental substance use behaviors (Foxcroft \& Lowe, 1991, 1995, 1997).

Generational similarities also manifest as similarities within social groups because SES is highly consistent across generations. Potentially, this helps to explain why SES is also associated with a range of health behaviors. If lower SES is associated with particular family socialization behaviors which, in turn, are associated with child risky behaviors, then we can regard family socialization as mediating the relation between SES and risky behavior (Singh-Manoux \& Marmot, 2005). For example, a family socialization deficit hypothesis predicts that lower SES is associated with poorer family socialization which, in turn, is linked to adolescent substance use.

Figure 1 sets out five typical alternative causal relations between family SES, family socialization, and young person substance use. Of these, most are plausible except for (Figure 1d) which suggests that family socialization practices are causally before family SES; this is less plausible as arguably SES (e.g., parental education) is a largely fixed characteristic that is not changed by later parenting practices. On the other hand, (Figure 1e) is much more plausible than (Figure 1d); it suggests that family SES is causally linked with family socialization practices and that both family socioeconomic status and family socialization practices are causally linked with substance use in young people. In other words, in (Figure 1e) family socialization mediates the relation between family socioeconomic status and Young Person Substance Use and can be expressed as a family socialization deficit hypothesis.

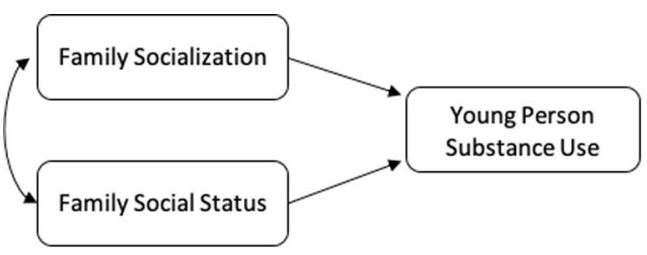

(a)

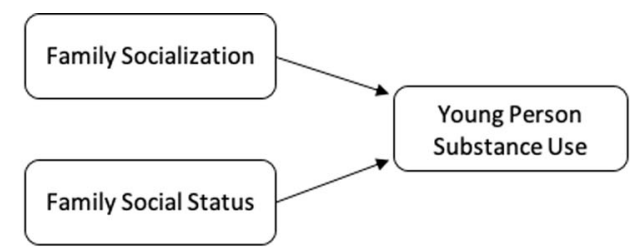

(b)

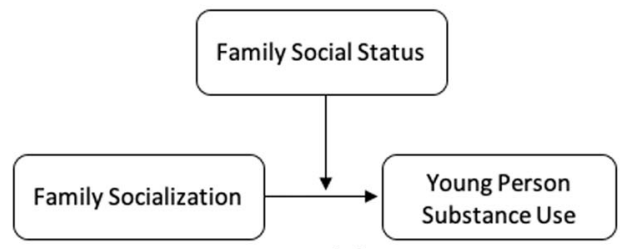

(c)

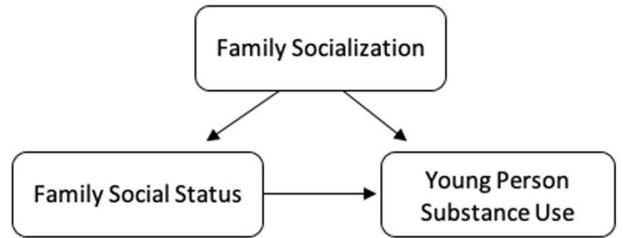

(d)

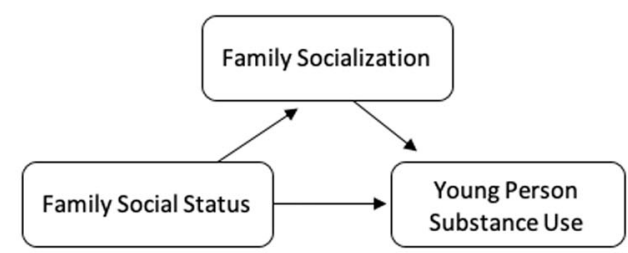

(e)

F I G U RE 1 (a) Family socialization covaries with family socioeconomic status, and both predict young person substance use; (b) family socialization is independent of family socioeconomic status, and both predict young person substance use; (c) family socioeconomic status moderates the relation between family socialization and young person substance use; (d) family socioeconomic status mediates the relation between family socialization and young person substance use; (e) family socialization mediates the relation between family socioeconomic status and young person substance user's 
One study drew conclusions that supported the family socialization deficit hypothesis (Hardaway \& Cornelius, 2014), finding that economic hardship was indirectly related to problem drinking in US adolescents through less supportive parenting (see, e.g., Figure 1e). However, this study only included families experiencing some form of economic hardship, and it may be that family socialization as a mediator is not so important with more advantaged families. This alternative explanation specifies that the relation between poor family socialization and risk behaviors is particularly salient for low SES families, that is, a stepped family socialization deficit hypothesis. In this scenario, SES is a moderator of the relation between family socialization and risk behaviors (Figure 1c). In terms of substance use, this would manifest as an important relation between family socialization and substance use initiation only for individuals from low-status families. In other words, this stepped family socialization deficit hypothesis predicts that SES moderates the relation between family socialization and substance use.

But intergenerational socialization is not the only socialization process. It sits alongside an intragenerational process (Singh-Manoux \& Marmot, 2005), where socioeconomic environment impacts dispositions and behavior more directly, for example through social class or group identity, work, or living conditions (Bourdieu, 1984). Material conditions and circumstances are important in their own right, contributing directly to the monotonic relationship between SES and health rather than being mediated or moderated through an intergenerational process. In this explanation, it is argued that individuals with lower status have lower levels of control over their lives, including work and economic situation, which directly leads to engagement in unhealthy behavior with associated poor health outcomes. For example, this status deficit hypothesis suggests that SES is directly predictive of substance use initiation and is not mediated by family socialization. Instead, family socialization may be independently predictive of substance use initiation (Figure 1a,b).

We specified several alternative hypotheses. H1 was expressed as the causal relation between SES and adolescent drinking being fully mediated by family socialization practices. $\mathrm{H} 2$ was expressed as the causal relation between family socialization variables and adolescent drinking being stronger for families with lower SES. This would be indicated by an interaction effect between SES and family socialization variables in the prediction of adolescent drinking. H3 was expressed as a direct and independent causal relation between SES and adolescent drinking, with no mediated effect. It is also plausible that we will find support for both mediated and direct effects of SES on adolescent risk behavior. This would be a partially mediated effect, that is, both mediated (indirect) and direct effects are statistically significant (H4).

\section{1 | Causal mediation analysis}

Traditional epidemiological analysis of the causal mechanism through which exposure is related to a health outcome has typically involved regression modeling to assess whether there is a relation between a mediator $(M)$ and an outcome $(Y)$ while controlling for an exposure $(\mathrm{X})$. If the size of $\mathrm{X} \rightarrow \mathrm{Y}$ diminishes or disappears once $\mathrm{M}$ is included in the model, then the effect of X on $\mathrm{Y}$ is described as being partially or fully mediated by M. For example (Pape et al., 2017), found that the relation between SES (measured by parental education) and adolescent drunkenness was nonsignificant once a set of family socialization variables were included in the regression model. They concluded that "The elevated risk of low SES vanishes when general parenting, alcohol-related parental permissiveness and parents' drinking are accounted for" (p. 792).

However, traditional approaches to mediation analysis are subject to two important limitations. The first problem is that the traditional approaches presuppose no interaction between the effects of the exposure and the mediator on the outcome. And the second problem is that, even if the exposure is randomized or if all the exposure-outcome confounders are included in the model, there may be unmeasured confounders of the mediator-outcome relationship. If control is not made for mediator-outcome confounders then results from the traditional approaches can be biased.

The causal inference approach to causal mediation analysis builds on the traditional approach by making these assumptions more explicit. The first assumption of no interaction between the effects of the exposure and the mediator on the outcome can be tested by including interaction terms in the model. If an interaction effect is present, then causal inference mediation analysis software can make adjustments for the interaction effect. The second assumption can be tested in software that estimates direct and indirect effects, even in the presence of unmeasured confounding, by allowing for nonlinear models and by advancing techniques for sensitivity analysis that examine robustness of effects to unmeasured confounding (Imai et al., 2010).

Specifically, the causal assumptions for the confounding that are made more explicit in the causal inference approach are (VanderWeele, 2016) as follows:

1. No unmeasured confounding of the exposure-outcome relation; that is, any variable that causes both the exposure and the outcome must be included in the model.

2. No unmeasured confounding of the mediator-outcome relation.

3. No unmeasured confounding of the exposure-mediator relation.

4. The exposure must not cause any confounder of the mediator-outcome relation. 
In the current study, we used the causal inference approach to test our alternative hypotheses in relation to specific indicators of SES, family socialization practices, and adolescent drinking behavior. To do this we have revisited a traditional mediation analysis (Pape et al., 2017) which did not report any checks on the causal assumptions required for robust causal inference, but did report that the elevated risk of low SES disappeared when general parenting, alcohol-related parental permissiveness and parental drinking mediators were accounted for. Traditional mediation analysis, for example, the different approach used in the analysis by Pape et al. (2017), potentially underestimates the direct effect conditioned on the mediators (Richiardi et al., 2013). Pape et al. (2017) implied a causal mechanism with the suggestion that measures to curb social inequality in adolescent drinking should target a broad range of parenting skills and practices. However, prevention efforts that target family socialization mediators in an attempt to address adolescent alcohol misuse and reduce alcoholrelated social disparities may be misplaced if the socialization mediation evidence does not hold up in the face of more robust causal analysis.

\section{2 | METHOD}

\subsection{Design}

The study employed a cross-sectional design, with print questionnaires completed by junior and high school students under supervision from teachers.

\subsection{Participants}

Data were collected in 2006 in 16 municipalities in Norway. Eighty-two out of 91 schools took part in the study, and the response rate at participating schools was $86 \%(N=17,761)$. Anonymized data were shared for the current analysis by the original researchers (Pape et al., 2017).

\section{$2.3 \quad$ Measures}

All measures were based on classroom completed adolescent self-reports.

\subsection{1 | Exposure: SES measures}

The primary indicator of SES was parents' education and a secondary indicator was marginal socioeconomic position (SEP). Parents' education was determined by the type of education received by the father in the first instance, or the mother if she was the primary or only caregiver. Educational categories included low (9 years of compulsory schooling or less), middle (high school), or high (college/university). Preliminary analysis indicated no substantive differences between participants with parents in the medium and high education categories, with regard to alcohol consumption. As such data were recoded into a dichotomous variable in which middle and high categories were coded as 0 , and low coded as 1 . Marginal SES was coded as 1 if one or more of the parents were in receipt of social welfare, or neither parent was in employment (part or full time). Other responses were coded as 0.

\subsection{2 | Mediators: Family socialization measures}

Family socialization variables included general parenting (three measures), alcohol-related parental permissiveness (two measures) and parents' drinking (two measures). All mediators were directionally coded such that putatively worse socialization practices (e.g., lower quality parent-child relationship, higher acceptance of child drinking, higher paternal drinking) had higher scores. In this way, all the mediators would (potentially) have positive signed correlations with the exposure and with the outcome measures. Parental knowledge and monitoring were assessed through four statements, which (Pape et al., 2017) reported as being adapted from (Wichstrøm, 2000), such as "my parents usually know where I am and what I do in my spare time." The quality of the parent-child relationship was evaluated using eight statements such as "I can talk to my parents about anything." Monitoring and relationship quality were measured on a four-point scale, ranging from "corresponds very poorly" (1) to "corresponds very well" (4), with Cronbach's $\alpha$ reported as acceptable for both measures (Pape et al., 2017). Scores were reversed for analysis so that higher scores indicated lower levels of monitoring and parent-child 
relationship quality. Experiences of lax parenting were estimated using three statements including being allowed to arrange unsupervised parties at home, staying out late in the evening without telling parents where they were, and having time limits for being out at night during the weekend. Yes/no responses were collected and summed, with a greater propensity for lax parenting indicated by higher scores.

Alcohol-related parental permissiveness included parental acceptance of drinking, evaluated using three statements, including "they do not want me to drink alcohol," "they would accept me drinking once in a while without getting drunk," and "they would accept that I got drunk once in a while." A four-point response scale was used and scores averaged. Cronbach's $\alpha$ was reported as acceptable (Pape et al., 2017). Higher scores indicated that parents were more accepting of their children drinking. Where adolescents reported consuming alcohol, parental permission to drink at home with peers was also assessed, using a single measure and no (coded as 0 ) and yes (coded as 1) response option.

Exposure to parental intoxication at least monthly was measured separately for maternal and paternal drinking using a four-point scale, ranging from never (0) to some times a week (4).

\subsubsection{Outcome: Substance use measures}

Two outcomes measures were any alcohol consumption, and any intoxication, in the past 12 months. Responses were coded 0 for no and 1 for yes.

\subsection{Analysis}

Code for the analysis is archived on the Open Science Framework (OSF) website (D.R. Foxcroft, 2021). We used R (R Core Team, 2019) and Mplus Muthén and Muthén (1998) for data analysis. Software versions used in the analysis are detailed in Supporting Information Appendix A. Analysis code for multiple mediator analysis was adapted from (Nguyen et al., 2016).

\subsection{1 $\quad$ Moderator analysis}

We addressed hypothesis $\mathrm{H} 2$ first because it was important to be able to assess any moderation (interaction) effects before proceeding with the mediation analysis. The $\mathrm{R}$ mediation package (Tingley et al., 2014) can assess the effect of interaction terms between the exposure and the mediator by adding this to the model and checking to see if the interaction term is significant. However, only one mediator at a time can be checked in this way (see Supporting Information Appendix $\mathrm{C}$ for illustrative analysis). Therefore, we used the R lme4 package (Bates et al., 2015) and specified a generalized linear mixed model (poisson log-link) with school as a random effect and with all exposure $\mathrm{x}$ mediator interaction terms included simultaneously.

\subsubsection{Mediator analysis}

If hypothesized mediators are conditionally independent, then separate causal mediation models can be fitted to assess the mediated effects through each of the mediators one at a time. However, with multiple mediators, the often implicit assumption that the different mediators are independent conditional on the exposure (and covariates) is a strong assumption. Moreover, it may be unrealistic, and when this assumption is violated it leads to biased estimates of causal effects (Tingley et al., 2014; VanderWeele \& Vansteelandt, 2014). When mediators are interdependent they can be thought of as being linked in a sequential causal chain, with a first mediator confounding the relation between a second mediator and the outcome and therefore violating the sequential ignorability assumption. ${ }^{1}$ An alternative approach is to combine interdependent mediators in a joint mediation approach, assuming a complete set of interdependent mediators such that other unmeasured confounding bias assumptions are not violated.

Importantly, this joint mediator approach does not necessarily require knowing the ordering of the mediators, and estimation of the combined effect of multiple mediators can be useful. We used the method from (Nguyen et al., 2016) to estimate the combined effect of multiple continuous/ordinal mediators, entered simultaneously with inter-correlations between all mediators, on a binary outcome using a structural equation model. An additional assumption is also included in this method: there is no mediator-mediator interaction in influencing the outcome measure, because such an interaction

\footnotetext{
${ }^{1}$ Sequential ignorability assumes that both the $\mathrm{X}$ to $\mathrm{M}$ and $\mathrm{X}$ to $\mathrm{Y}$ relationships are not confounded, and also that the $\mathrm{M}$ to $\mathrm{Y}$ relationship is not confounded.
} 
could result in error combinations being non-normal, due to a product of the mediators' error terms. Their method estimates potential outcome probabilities, and natural direct and indirect effects using the causal inference potential outcomes framework. Alongside natural direct and indirect effects, the controlled direct effect may be useful for interpretation: this corresponds to the exposure effect that would remain after an intervention that sets the mediator(s) to a specific level (see [Richiardi et al., 2013] for definitions of natural direct and indirect, as well as controlled direct, effects in epidemiological studies). The controlled direct effect has been suggested to be more useful in observational research (Richiardi et al., 2013), particularly research involving health disparities (Naimi et al., 2014). Importantly, if there is no exposure-mediator interaction then the controlled direct effect and the natural direct effect are equivalent (Richiardi et al., 2013), and in our analysis, the specific level that the mediator(s) would be set is to the non-exposed level.

\subsection{3 | Sensitivity analysis}

Sensitivity analysis is used to check how susceptible a mediated effect is to the risk of confounding. While all four of the previously mentioned confounding assumptions are relevant for interpreting causality with natural direct and indirect effects, only the first two assumptions are relevant for interpreting causality with controlled direct effects. Formal sensitivity analysis methods have focused on the mediator-outcome relation for single mediators, and for a two mediator sequential effect, in the R mediation package (Tingley et al., 2014). See Supporting Information Appendix E, for examples. However, formal sensitivity analysis for more than two mediators in a joint mediation approach is not currently available, although it has been identified as an area for future work (Nguyen et al., 2016). Therefore, in the absence of a formal calculated sensitivity analysis parameter such as rho, an interpretive judgment based on the size of the mediated effect, the completeness of the joint set of mediators, and the likelihood of other potential unmeasured confounders, can be made in the joint mediation approach. To assist this judgment, we used the $E$ value which provides an approximate sensitivity analysis for different types of unmeasured confounding (Smith \& VanderWeele, 2019; VanderWeele \& Ding, 2017).

\subsubsection{Ethics}

The original study was approved by the Norwegian Social Science Data Services. The current study is based on analyses of anonymized secondary data, and no further ethical approval was required.

\section{RESULTS}

Participants were 17,761 young people aged $13-18$ years (48.8\% male; $50.6 \%$ female). The proportions of adolescents within each age group were broadly similar. Less than $10 \%$ of the sample came from families where the parents had lower levels of education (6.8\%) or were receiving financial aid (8.5\%). Across the whole sample, 10,170 (57.3\%) had consumed alcohol and 7779 (43.8) reported being intoxicated in the past year (Table 1).

\section{1 | Replication analysis}

We first replicated the original traditional mediation analysis using the difference method reported by (Pape et al., 2017). Limiting the sample to respondents aged 13-16, as per the original analysis, our results were consistent with the previous findings (Supporting Information Appendix B). For example, in our analysis, we estimated the relative risk (RR) for the Base model, with Drinker prevalence as the outcome and Parental Education as the exposure, as 1.21 (1.10-1.34) which was consistent with the estimate from (Pape et al., 2017) of 1.21 (1.13-1.29). For the full model, including family socialization mediators, the estimates were also consistent: $0.99(0.90-1.10)$ and 1.00 (0.94-1.06), respectively.

\section{2 $\mid$ Moderation analysis}

Using the full sample (all age groups) we found that, overall, there was no evidence for interaction effects in the prediction of the outcome. This indicates that the family socialization effects on the outcome are consistent across different values of the exposure (Table 2). Parental acceptance of drinking may be moderated very slightly, only for the parental education exposure, as this was significant at $p<.05$, but this could be a false positive given the number of tests. This analysis does not 
T A B LE 1 Sample demographic information, by gender

\begin{tabular}{|c|c|c|c|c|c|c|c|c|}
\hline \multirow[b]{2}{*}{ Age } & \multirow[b]{2}{*}{13} & \multicolumn{2}{|l|}{ Male } & \multicolumn{2}{|c|}{ Female } & \multicolumn{2}{|c|}{ Missing } & \multirow{2}{*}{$\begin{array}{r}\text { Totals } \\
2829\end{array}$} \\
\hline & & 1381 & $48.8 \%$ & 1430 & $50.5 \%$ & 18 & $0.6 \%$ & \\
\hline & 14 & 1473 & $49.6 \%$ & 1474 & $49.7 \%$ & 21 & $0.7 \%$ & 2968 \\
\hline & 15 & 1571 & $49.3 \%$ & 1584 & $49.7 \%$ & 29 & $0.9 \%$ & 3184 \\
\hline & 16 & 1753 & $51.1 \%$ & 1672 & $48.8 \%$ & 4 & $0.1 \%$ & 3429 \\
\hline & 18 & 936 & $43.5 \%$ & 1204 & $55.9 \%$ & 13 & $0.6 \%$ & 2153 \\
\hline \multirow[t]{3}{*}{ Parent education } & High & 3834 & $47.8 \%$ & 4142 & $51.6 \%$ & 45 & $0.6 \%$ & 8021 \\
\hline & Medium & 4266 & $50.0 \%$ & 4222 & $49.5 \%$ & 47 & $0.6 \%$ & 8535 \\
\hline & Low & 574 & $47.6 \%$ & 626 & $52.0 \%$ & 5 & $0.4 \%$ & 1205 \\
\hline Past year drinking & Yes & 4688 & $46.1 \%$ & 5426 & $53.4 \%$ & 56 & $0.6 \%$ & 10170 \\
\hline \multirow[t]{2}{*}{ Past year drunkenness } & No & 5160 & $51.7 \%$ & 4764 & $47.7 \%$ & 58 & $0.6 \%$ & 9982 \\
\hline & Yes & 3514 & $45.2 \%$ & 4226 & $54.3 \%$ & 39 & $0.5 \%$ & 7779 \\
\hline
\end{tabular}

Note: Overall $N=17,661$.

provide support for $\mathrm{H} 2$, the stepped family socialization deficit hypothesis and therefore, in the causal mediation analysis, we excluded interaction terms.

\section{3 | Causal mediation analysis with multiple mediators}

Potential outcomes were estimated for each model. For example, focusing on the model for the parental education exposure with the drunkenness prevalence outcome, the potential prevalence of adolescent self-reported yearly drunkenness had the whole sample been in the medium/high parental education condition was estimated to be $00=32.2 \%$; and had the whole sample been in the low parental education condition the potential prevalence was estimated to be p11 $=42.8 \%$. Had the whole sample been in the low parental education condition but the mediators kept at the medium/high parental education levels, then the potential prevalence of yearly drunkenness was estimated to be p10 $=34.1 \%$. Conversely, had the whole sample been in the medium/high parental education condition but the mediators kept at the low parental education levels, then the potential prevalence of yearly drunkenness was estimated to be p $01=40.8 \%$. These four potential outcome estimates ( $\mathrm{p} 00, \mathrm{p} 11, \mathrm{p} 10$, and $\mathrm{p} 01$ ) were used to calculate risk difference (RD) and RR estimates for total, natural indirect, and natural direct effects, and these are shown in Table 3. As well as the models for the full sample, we also provide results by age groups 13-14, 15-16, and 17-18 in Figure 2 and Supporting Information Appendix D.

Overall, the estimates are slightly stronger for the parental education exposure than for the marginal SEP exposure. The effect of both exposures on either drinking or drunkenness over the past year is fully mediated, supporting H1. For the full sample, on the $\mathrm{RD}$ (the difference between the risk of the outcome in the exposed group and the unexposed group) scale the natural indirect effect estimates ranged from 5.4\% for the Drunk Marginal SEP model to 9.8\% for the Drinker Parental Education model. In contrast, the natural direct effect RD estimates range from $2.7 \%$ for the Drunk $\sim$ Marginal SEP model to $1.2 \%$ for the Drinker Parental Education model. On the RR (the multiplicative increase (or decrease) in the risk of the outcome in the exposed group compared to the unexposed group) scale the natural indirect effect estimates, with $95 \%$ confidence intervals, ranged from $1.13(1.10-1.17)$ for the Drinker Marginal SEP model to $1.27(1.21-1.32)$ for the Drunk Parental Education model. In contrast, the natural direct effect RR estimates ranged from 1.02 (0.97-1.07) for the Drinker Marginal SEP model to 1.07 (1.00-1.14) for the Drunk Parental Education model.

These results clearly provide support for $\mathrm{H} 1$, the family socialization deficit hypothesis. By definition, as $\mathrm{H} 1$, the fully mediated model, is clearly supported by the results, we can also report that $\mathrm{H} 3$, the status deficit hypothesis, and $\mathrm{H} 4$, the partial mediation hypothesis, are not supported. 
T A B LE 2 Moderation model parameter estimates (95\% confidence intervals) for different outcome (drinker or drunkenness) and socioeconomic status (parental education or marginal socioeconomic position, SEP) variables

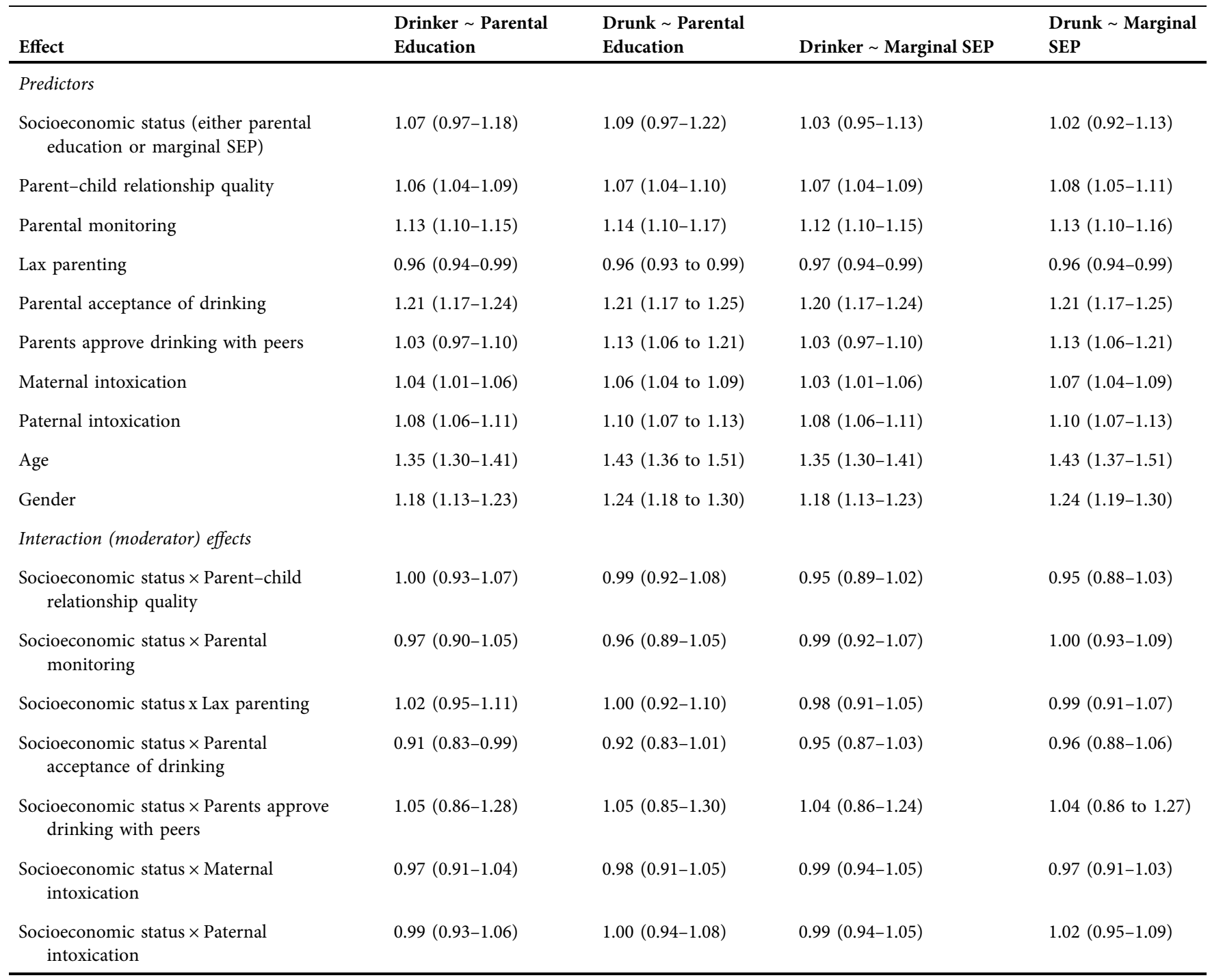

Note: Moderation model with complete sample of 13- to 18-year-olds, $N=17,493$ included in model; the symbol “ ” is notation used in the R programming language to separate the dependent variable (drinking) from the independent variable (socioeconomic status).

In a subgroup analysis, by age, the natural indirect effects are particularly strong for the youngest age group, with a RR of $1.90(1.60-2.24)$ for the Drunk Parental Education model in 13- to 14-year-olds (Figure 2 and Supporting Information Appendix D). The natural indirect effect is weaker for 15- to 16-year-olds, and practically disappears amongst the oldest group, with a RR of 1.06 (1.03-1.10) for the Drunk Parental Education model in 17- to 18-year-olds. Although we have not made a formal statistical comparison across age groups, the subgroup results may suggest that $\mathrm{H} 1$, the family socialization deficit hypothesis, is particularly salient for younger adolescents, but is much less relevant as young people move into their later teenage years.

If the model is correctly specified and the identifying assumptions hold, we can interpret these effects as causal. For example, in the Drunk Parental Education model for the subsample of 15- to 16-year-olds, if the whole subsample had been in the low parental education category (as opposed to medium/high parental education), this would have increased annual drunkenness prevalence by $13.3 \%$ (the total effect). If the whole 15- to 16-year-old subsample had been in the low parental education category, but the mediators had been kept at medium/high parental education levels and not allowed to change, this hypothetical condition would have increased annual drunkenness prevalence, relative to the medium/high parental education category, by only a small amount: $2.7 \%$ (the direct/unmediated effect). Relative to this hypothetical condition, if the whole 15- to 16-year-old subsample had been in the low education category and their mediators were kept at levels consistent with low parental education, then annual drunkenness prevalence would be increased by $12.8 \%$ (the indirect/ mediated effect). 


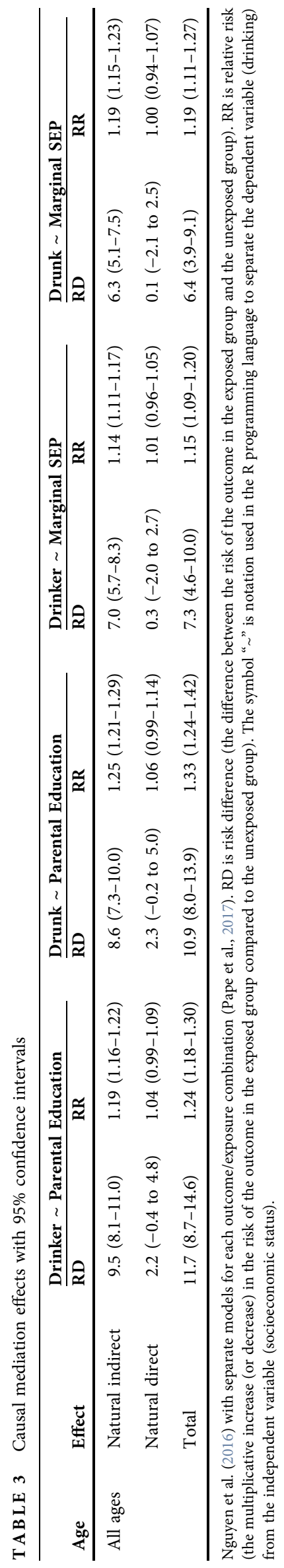



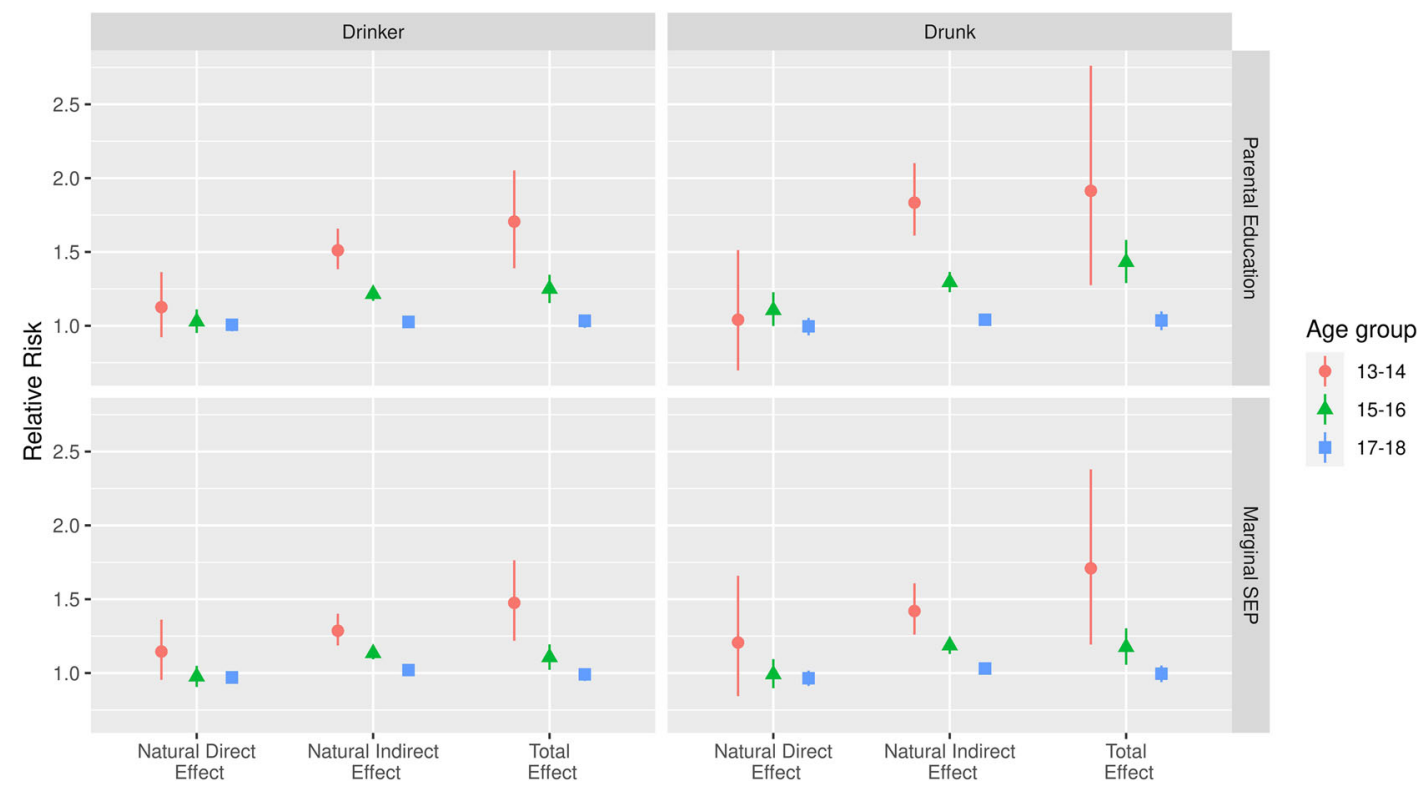

F IG URE 2 Age subgroup causal mediation effects with 95\% confidence intervals for different outcomes (drinker status, drunk status) and exposure (parental education, marginal socioeconomic position [SEP]) multiple mediator models and effects specified as relative risks. See Supporting Information Appendix D for full table of effect estimates [Color figure can be viewed at wileyonlinelibrary.com]

\subsection{Sensitivity analysis for confounding}

In a formal sensitivity analysis for single mediators, the value of the mediated effect is much lower than with the multiple mediator set reported in Table 3. Linked to this, the sensitivity analysis parameter, rho, is small (see Supporting Information Appendix E) and indicates that any unmeasured confounding bias could easily remove the natural indirect effect. However, the natural indirect effect with the multiple mediator set is much larger, and therefore it is somewhat less likely that unmeasured confounding bias would eliminate the natural indirect effect. In approximate sensitivity analysis, the RR $E$ value ${ }^{2}$ was 1.51 (lower bound $[\mathrm{lb}]=1.28$ ) for the risk of confounding between the exposure and the outcome (no confounding Assumption 1), and $2.00(\mathrm{lb}=1.89)$ for the risk of confounding between the mediator and the outcome (no confounding Assumption 2), in the Drunk Parental Education model (effect RRs from Supporting Information Appendix B and D, respectively). In the youngest age-group, the $E$ values were $3.02(\mathrm{lb}=2.04)$ (Assumption 1$)$, and $3.36(\mathrm{lb}=2.87)$ (Assumption 2). The $E$ value can be interpreted as the effect size that unmeasured confounders would have to reach to fully account for the observed effect; specifically, it is the joint minimum strength of association on the risk ratio scale that an unmeasured confounder must have with the treatment and outcome to fully explain away an observed treatment-outcome risk (VanderWeele \& Ding, 2017).

\section{DISCUSSION}

In this analysis, we revisited a traditional mediation analysis using a causal mediation approach, and the hypothesis that SES effects on adolescent substance abuse are fully mediated by family socialization practices was supported in our modeling. The natural indirect effects in the multiple mediator analysis were consistent across different exposure and outcome measures and were relatively stronger in younger age groups. In our causal mediation analysis, the natural direct effect fixed the combined mediators for each individual to the level which they naturally would have been under the absence of exposure. The natural indirect effect assumes that exposure is set to some level (e.g., "nonexposed") and then compares what would have happened if the combined mediators were set to "nonexposed" versus "exposed." While this analysis is appropriate from a technical perspective, it is not so straightforward to interpret from a policy perspective (Naimi et al., 2014). It typically is not practical to put in place a policy that will switch people from being "exposed" to a "nonexposed" status (unlike in intervention trials where a switch from "not treated" to "treated" is entirely plausible). So for epidemiological studies natural direct and natural indirect effects have a less straightforward interpretation as they cannot easily be used to understand the change in the

${ }^{2} \mathrm{R}$ code for the calculated $E$ values is in the OSF archive (D. R. Foxcroft, 2021). 
outcome that might be achieved by intervening on the exposure and the mediator(s). Instead, the controlled direct effect may be useful for interpretation: this corresponds to the exposure effect that would remain after an intervention that sets the mediator(s) to a specific level. As we found no exposure-mediator interaction then the controlled direct effect and the natural direct effect are equivalent, and in our analysis, the specific level that the mediator(s) would be set to is the nonexposed level. For example, we can report that if an intervention changed family socialization practices such that everyone was brought up to the higher levels shown in the medium/high parental education (the nonexposed) group, then disparities in adolescent drunkenness prevalence amongst 15 - to 16 -year-olds would be reduced to $2.0 \%$.

\section{1 $\quad$ Limitations}

The analysis in this paper relies on cross-sectional data, as all measures were collected at one point in time. This does limit the causal inferences that can be made, although we suggest that SES measures are largely fixed and stable, that family socialization is generally consistent within families over time, and that it is unlikely that family socialization patterns or adolescent alcohol use cause SES, for example, level of parental education. That said, we have relied on self-reported survey data so this may introduce some self-report bias, and also the possibility that family socialization and adolescent drinking are interdependent. There is some evidence that survey measures of parenting and outcomes are dynamically and dyadically determined by youth and parents (Bell \& Chapman, 1986; Lytton, 2000; Wilkinson et al., 2021).

The outcome in our analysis was either drinking or drunkenness prevalence, but alcohol use may not be as closely linked to SES as other health behaviors. Furthermore, this relation may be contingent on the SES measure used. There is some evidence that low socioeconomic status adolescents report poorer nutritional habits, less exercise as well as smoking, but limited evidence for alcohol consumption or marijuana use (Hanson \& Chen, 2007). In particular, it appeared that lower SES may be more likely to be related to negative health behaviors if SES was measured using SES indicators such as parent education. In contrast, there was some support to suggest that, when indicators of family financial resources are used, higher SES adolescents are at greater risk for marijuana use.

In our causal mediation analysis, there was no substantive or consistent evidence of exposure-mediator interactions, and therefore this assumption was met. We have also stated that the controlled direct effect is more relevant for observational studies, especially studies of health disparities, and therefore, only the first two assumptions of no confounding (the exposure-outcome relation and the mediator-outcome relation) are relevant for causal interpretation. In our analysis, we did not include an extensive set of potential confounders because of limited data, and it is possible that unmeasured confounding of the exposure-outcome relation could account for the mediation effect that we found. For example, it is plausible that other variables such as parental psychopathology (e.g., depression), genotype, local economic conditions could confound the results. We acknowledge that mediational causal inference in observational studies is fraught with difficulty, some might say it is an alchemic quest. As others have previously noted, mediation analysis is not able to identify unique mediators, nor distinguish between alternative causal models (Fiedler et al., 2011). What we also do not know from this analysis is how the different mediators contribute to the mediation effect, and therefore how they might each be targeted through policy or interventions. This is a limitation of the causal inference joint mediator approach (Nguyen et al., 2016).

\section{5 | CONCLUSION}

We were able to replicate the results from (Pape et al., 2017) and move toward a more formal causal interpretation. The results provide some tentative causal evidence support for the idea that improving family socialization practices could potentially reduce risk behaviors and improve health outcomes, especially with a universal approach, or even a proportionate universal approach (M. Marmot, 2010) may help reduce health disparities. This evidence was stronger in the younger 13- to 14-year-old age group. However, we were not able to fully account for the potential risk of confounding either in study design, modeling, or sensitivity analysis. That said, other recent studies also support the idea of improving family socialization practices. For example (Chen et al., 2019), concluded that positive parenting improves multiple aspects of health and well-being in young adulthood. Effective parent and family interventions might usefully contribute as part of a comprehensive prevention system that includes macrolevel policies to tackle the structural determinants of health disparities, such as poverty, alongside, family and individual approaches (Foxcroft, 2014; Pearce et al., 2019).

As the next step for future research, other studies and analyses could check the consistency of findings across settings and measures, especially as substance use initiation is probably not a straightforward measure of health and well-being. Our findings suggest it is especially important to undertake multiple mediator analysis with a theoretically informed and complete set of mediators, and future research should also investigate the contribution of individual mediators in a multiple mediator analysis while adjusting for a range of potential confounders. And further intervention development and evaluation studies 
are required to advance understanding of why some family interventions appear to be effective in some settings and contexts, but less so in others (Gilligan et al., 2019).

\section{1 | Data, materials, and online resources}

The code to reproduce these analyses is available at https://osf.io/u49aw/?view_only-81e2df88ae5444739dc14c0d97331f84. The OSF project contains all necessary files to reproduce the analysis in MPlus and R, as well as a preprint of this manuscript. Requests for data sharing can be made to Pape et al. (2017) who shared the data for the analysis presented in this paper.

\section{CONFLICT OF INTERESTS}

The authors declare no conflict of interest.

\section{AUTHOR CONTRIBUTIONS}

Study conceptualization, statistical programming, and analysis, drafted initial and final sections of the introduction, results, and discussion sections: David R. Foxcroft. Main contributors to data preparation and method section: Sarah J. Howcutt and Fiona Matley. Main contributor to the description of alternative causal mechanisms in the introduction: Louise Taylor Bunce. Study conceptualization and development of the framing of the introduction and discussion in relation to other literature: Emma L. Davies. All authors contributed to manuscript conceptualization, editing, and review for submission.

\section{ORCID}

David R. Foxcroft (D) http://orcid.org/0000-0001-9752-7527

Sarah J. Howcutt (D) http://orcid.org/0000-0002-4844-1732

Fiona Matley (D) http://orcid.org/0000-0001-5322-2852

Louise Taylor Bunce (D) http://orcid.org/0000-0002-8754-9957

Emma L. Davies (D) http://orcid.org/0000-0003-3577-3276

\section{PEER REVIEW}

A preprint version of this manuscript before peer-review can be found on OSF: https://osf.io/u49aw/(version 1)

\section{REFERENCES}

Bates, D., Mächler, M., Bolker, B., \& Walker, S. (2015). Fitting linear mixed-effects models using lme4. Journal of Statistical Software, 67(1), 1-48. https://doi. org/10.18637/jss.v067.i01

Bell, R. Q., \& Chapman, M. (1986). Child effects in studies using experimental or brief longitudinal approaches to socialization. Developmental Psychology, 22(5), 595-603. https://doi.org/10.1037/0012-1649.22.5.595

Bourdieu, P. (1984). Distinction. Routledge.

Cablova, L., Pazderkova, K., \& Miovsky, M. (2014). Parenting styles and alcohol use among children and adolescents: A systematic review. Drugs-Education Prevention and Policy, 21, 1-13.

Chen, Y., Haines, J., Charlton, B. M., \& VanderWeele, T. J. (2019). Positive parenting improves multiple aspects of health and well-being in young adulthood. Nature Human Behaviour, 3, 684-691. https://doi.org/10.1038/s41562-019-0602-x

Fiedler, K., Schott, M., \& Meiser, T. (2011). What mediation analysis can (not) do. Journal of Experimental Social Psychology, 47(6), 1231-1236. https://doi org/10.1016/j.jesp.2011.05.007

Foxcroft, D. R. (2014). Fit for purpose: A form and function-based taxonomy for prevention is arguably more refined, accurate, and predictive. Prevention Science, 15(6), 829-830.

Foxcroft, D. R. (2021, October 25). Mediation. https://doi.org/10.17605/OSF.IO/U49AW

Foxcroft, D. R., \& Lowe, G. (1991). Adolescent drinking behaviour and family socialization factors: A meta-analysis. Journal of Adolescence, 14(3), 255-273. https://doi.org/10.1016/0140-1971(91)90020-R

Foxcroft, D. R., \& Lowe, G. (1995). Adolescent drinking, smoking and other substance use involvement: Links with perceived family life. Journal of Adolescence, 18(2), 159-177.

Foxcroft, D. R., \& Lowe, G. (1997). Adolescents' alcohol use and misuse: The socializing influence of perceived family life. Drugs-Education Prevention and Policy, 4(3), 215-229.

Gilligan, C., Wolfenden, L., Foxcroft, D. R., Williams, A. J., Kingsland, M., Hodder, R. K., Stockings, E., McFadyen, T.-R., Tindall, J., Sherker, S., Rae, J., \& Wiggers, J. (2019). Family-based prevention programmes for alcohol use in young people. Cochrane Database of Systematic Reviews, 3, 3. https://doi. org/10.1002/14651858.CD012287.pub2

Hanson, M. D., \& Chen, E. (2007). Socioeconomic status and health behaviors in adolescence: A review of the literature. Journal of Behavioral Medicine, 30(3), 263-285. https://doi.org/10.1007/s10865-007-9098-3

Hardaway, C. R., \& Cornelius, M. D. (2014). Economic hardship and adolescent problem drinking: Family processes as mediating influences. Journal of Youth and Adolescence, 43(7), 1191-1202. https://doi.org/10.1007/s10964-013-0063-x

Imai, K., Keele, L., \& Tingley, D. (2010). A general approach to causal mediation analysis. Psychological Methods, 15(4), 309-334. https://doi.org/10.1037/ a0020761

Lytton, H. (2000). Toward a model of family-environmental and child-biological influences on development. Developmental Review, 20(1), 150-179. https:// doi.org/10.1006/drev.1999.0496 
Marmot, M. (2005). Social determinants of health inequalities. Lancet, 365(9464), 1099-1104. https://doi.org/10.1016/S0140-6736(05)71146-6

Marmot, M. (2010). Fair society, healthy lives: The Marmot review. Strategic review of health inequalities in England post, 2010.

Marmot, M. G. (2004). The status syndrome: How social standing affects our health and longevity (1st American). Times Books.

Muthén, L. K., \& Muthén, B. O. (1998). Mplus user's guide (8th ed.). Muthén \& Muthén.

Naimi, A. I., Kaufman, J. S., \& MacLehose, R. F. (2014). Mediation misgivings: Ambiguous clinical and public health interpretations of natural direct and indirect effects. International Journal of Epidemiology, 43(5), 1656-1661. https://doi.org/10.1093/ije/dyul07

Nguyen, T. Q., Webb-Vargas, Y., Koning, I. M., \& Stuart, E. A. (2016). Causal mediation analysis with a binary outcome and multiple continuous or ordinal mediators: Simulations and application to an alcohol intervention. Structural Equation Modeling: A Multidisciplinary Journal, 23(3), 368-383. https:// doi.org/10.1080/10705511.2015.1062730

Pape, H., Norström, T., \& Rossow, I. (2017). Adolescent drinking: A touch of social class? Addiction, 112(5), 792-800. https://doi.org/10.1111/add.13721

Pearce, A., Dundas, R., Whitehead, M., \& Taylor-Robinson, D. (2019). Pathways to inequalities in child health. Archives of Disease in Childhood, 104, 998-1003. https://doi.org/10.1136/archdischild-2018-314808

R Core Team. (2019). R: A Language and Environment for Statistical Computing. R Foundation for Statistical Computing. https://www.R-project.org/

Richiardi, L., Bellocco, R., \& Zugna, D. (2013). Mediation analysis in epidemiology: Methods, interpretation and bias. International Journal of Epidemiology, 42(5), 1511-1519. https://doi.org/10.1093/ije/dyt127

Singh-Manoux, A., \& Marmot, M. (2005). Role of socialization in explaining social inequalities in health. Social Science \& Medicine, 60(9), $2129-2133$. https://doi.org/10.1016/j.socscimed.2004.08.070

Smith, L. H., \& VanderWeele, T. J. (2019). Mediational E-values: Approximate sensitivity analysis for unmeasured mediator-outcome confounding. Epidemiology (Cambridge, Mass.), 30(6), 835-837. https://doi.org/10.1097/EDE.0000000000001064

Tingley, D., Yamamoto, T., Hirose, K., Keele, L., \& Imai, K. (2014). Mediation: R package for causal mediation analysis. Journal of Statistical Software, 59(5), 1-38. https://doi.org/10.18637/jss.v059.i05

VanderWeele, T. J. (2016). Mediation analysis: A practitioner's guide. Annual Review of Public Health, 37(1), 17-32. https://doi.org/10.1146/annurevpublhealth-032315-021402

VanderWeele, T. J., \& Ding, P. (2017). Sensitivity analysis in observational research: Introducing the E-value. Annals of Internal Medicine, 167(4), $268-274$. https://doi.org/10.7326/M16-2607

VanderWeele, T. J., \& Vansteelandt, S. (2014). Mediation analysis with multiple mediators. Epidemiologic Methods, 2(1), 95-115. https://doi.org/10.1515/em2012-0010

Wichstrøm, L. (2000). Psychological and behavioral factors unpredictive of disordered eating: A prospective study of the general adolescent population in Norway. The International Journal of Eating Disorders, 28(1), 33-42. https://doi.org/10.1002/(sici)1098-108x(200007)28:1\%3C33::aid-eat5\%3E3.0. $\mathrm{co} ; 2-\mathrm{h}$

Wilkinson, K., Ball, S., Mitchell, S. B., Ukoumunne, O. C., O'Mahen, H. A., Tejerina-Arreal, M., Hayes, R., Berry, V., Petrie, I., \& Ford, T. (2021). The longitudinal relationship between child emotional disorder and parental mental health in the British Child and Adolescent Mental Health surveys 1999 and 2004. Journal of Affective Disorders, 288, 58-67. https://doi.org/10.1016/j.jad.2021.03.059

\section{SUPPORTING INFORMATION}

Additional supporting information may be found in the online version of the article at the publisher's website.

How to cite this article: Foxcroft, D. R., Howcutt, S. J., Matley, F., Taylor Bunce, L., \& Davies, E. L. (2022). Testing socioeconomic status and family socialization hypotheses of alcohol use in young people: A causal mediation analysis. Journal of Adolescence, 94, 240-252. https://doi.org/10.1002/jad.12023 